\title{
Pharmacological Properties of Linearolactone against the Amoebiasis Caused by Entamoeba histolytica: An In Silico Study ${ }^{\dagger}$
}

\author{
Luis Varela-Rodríguez ${ }^{1, *, \ddagger(\mathbb{D})}$, José Antonio Velázquez-Domínguez ${ }^{2, *, \ddagger \mathbb{D}}$, Verónica Ivonne Hernández-Ramírez ${ }^{2}$ (D), \\ Hugo Varela-Rodríguez ${ }^{1}{ }^{(\mathbb{D}}$, Audifas Salvador Matus-Meza $^{3}$, Fernando Calzada ${ }^{4}$ D, Elihu Bautista ${ }^{5}$ \\ and Patricia Talamás-Rohana $2, *$ (D)
}

check for updates

Citation: Varela-Rodríguez, L.; Velázquez-Domínguez, J.A.; Hernández-Ramírez, V.I.; Varela-Rodríguez, H.; Matus-Meza, A.S.; Calzada, F.; Bautista, E.;

Talamás-Rohana, P. Pharmacological Properties of Linearolactone against the Amoebiasis Caused by Entamoeba histolytica: An In Silico Study. Med. Sci. Forum 2021, 7, 3. https:// doi.org/10.3390/ECMS2021-10843

Academic Editor: Silvia Selleri

Published: 31 August 2021

Publisher's Note: MDPI stays neutral with regard to jurisdictional claims in published maps and institutional affiliations.

Copyright: (C) 2021 by the authors. Licensee MDPI, Basel, Switzerland. This article is an open access article distributed under the terms and conditions of the Creative Commons Attribution (CC BY) license (https:// creativecommons.org/licenses/by/ $4.0 /)$
1 Facultad de Enfermería y Nutriología, UACH, Chihuahua CP 31125, Mexico; hvrodriguez@uach.mx

2 Departamento de Infectómica y Patogénesis Molecular, CINVESTAV-IPN, Ciudad de México CP 07360, Mexico; arturomvi@hotmail.com

3 Departamento de Farmacia-Facultad de Química, UNAM, Ciudad de México CP 04510, Mexico; audi_matus@hotmail.com

4 Unidad de Investigación Médica en Farmacología, Hospital de Especialidades UMAE-CMNSXXI-IMSS, Ciudad de México CP 06725, Mexico; fercalber10@gmail.com

5 Unidad de Ciencias Ambientales, IPICYT, San Luis Potosí CP 78216, Mexico; hormigaqfb@hotmail.com

* Correspondence: lvrodriguez@uach.mx (L.V.-R.); jauam14@yahoo.com.mx (J.A.V.-D.); ptr@cinvestav.mx (P.T.-R.)

+ Presented at the 1st International Electronic Conference on Molecular Sciences: Druggable Targets of Emerging Infectious Diseases (ECMS 2021), 1-14 September 2021; Available online: https:/ / ecms2021.sciforum.net/.

$\ddagger$ These authors contributed equally to this work.

Abstract: Linearolactone (LL) isolated from Salvia polystachya presents antiparasitic activity against E. histolytica and G. lamblia through ROS production, an apoptosis-like process, and alteration of the actin cytoskeleton. This effect limits the invasion and spread of parasites during host infection. However, the possible toxicological effects or the molecular mechanisms by which LL affects the E. histolytica mobility are still not understood. LL could act as an inhibitor of accessory cytoskeletal proteins, such as myosin, calreticulin, and calpain to achieve this end. The aim of this study was to determine the pharmacological and toxicological properties of LL via bioinformatic analyses to find therapeutic targets and to understand the action mechanism on the actin cytoskeleton against E. histolytica. The pharmacological activities, toxicological risks, and molecular targets of LL were determined using free software such as Molsoft(c) to define the bioactivity through comparison with standard drugs [1], Molinspiration (C) to calculate physicochemical properties [2], ToxiM@ to determine possible intestinal permeability [3,4], SuperCYPsPred@ to predict drug metabolism via the cytochrome-P450 system [5,6], and SEAC to find proteins with binding sites for the active compounds through an inverse protein-ligand approach $[7,8]$. Molecular docking with key proteins for the pathogenic activity of E. histolytica trophozoites, such as myosin-II and calreticulin, was performed with AutoDock-Vina and UCSF-Chimera. Results revealed that LL presents a druglikeness of -0.55 and ToxiM of 0.958 due to medium toxicity associated with interactions in nuclear receptors (0.66), GPCR ligands (0.65), and enzymatic inhibitions (0.47) related to the cytochromeP450 system (CYP3A4, low). Results indicate that LL is a hydrophobic molecule (LogP: 1.59) with intermediate intestinal absorption (TPSA: 65.75, CACO-2 permeability) and medium blood-brain barrier penetration (3.86). SEA analysis demonstrated that the potential target pharmacophores are OPRK1 ( $p$-Value: $6.49 \times 10^{-37}$, Max TC: 0.49) and NLRP3 ( $p$-Value: $3.90 \times 10^{-19}$, Max TC: 0.36) in humans. Molecular docking of LL with E. histolytica proteins showed high affinity to ATP-binding catalytic sites in the heavy-chain (GLU-187.A, THR-186.A, ASN-234.B) of myosin-II $(-8.30 \mathrm{Kcal} / \mathrm{mol})$, as well as in chain A and C (LYS-199.A, LYS-152.C) of calreticulin $(-8.77 \mathrm{Kcal} / \mathrm{mol})$. As for conclusions, LL is a compound with possible moderate toxicity, sedative effects on CNS, and anti-inflammatory properties. In addition, LL has antiparasitic activity involving the immobilization of E. histolytica trophozoites through interactions with accessory proteins from the actin cytoskeleton 
such as myosin-II and calreticulin. These proteins are present in the parasite and are fundamental to amoebic liver abscess formation during host infection. Therefore, LL could be a therapeutic alternative to the amoebiasis treatment and provide a leading compound for drug discovery against parasitic diseases, but in-depth studies are necessary to confirm these claims.

Keywords: Linearolactone; pharmacological properties; toxicological effects; Entamoeba histolytica; in silico analysis

Supplementary Materials: The poster presentation is available online at https:/ / www.mdpi.com/ article/10.3390/ECMS2021-10843/s1.

Funding: This work was supported by the Basic Science CONACyT-Project (grant number CB-201301-221136) to P.T.-R.; L.V.-R. was a recipient of a Ph.D. scholarship (Reg. 369164), and J.A.V.-D. was a recipient of a Postdoctoral fellowship (Reg. 103584) from the Consejo Nacional de Ciencia y Tecnología (CONACyT, México).

Institutional Review Board Statement: Not applicable.

Informed Consent Statement: Not applicable.

Data Availability Statement: All data generated or analyzed during this study are included in this published article (as well as supplementary information files). Raw data are available from the corresponding author on reasonable request.

Acknowledgments: The authors thank to Diana Laura Pichardo-Hernández, Mayra Herrera-Martínez, Rodrigo Daniel Castellanos-Mijangos, and Bibiana Chávez-Munguía for the participation and interest in this project; Daniel Morales-Mora and Juan Carlos Osorio-Trujillo for their technical support.

Conflicts of Interest: The authors declare no conflict of interest. The funders had no role in the design of the study; in the collection, analyses, or interpretation of data; in the writing of the manuscript, or in the decision to publish the results.

\section{References}

1. Molsoft LLC. Drug-Likeness and Molecular Property Prediction. 2021. Available online: http://molsoft.com/mprop/ (accessed on 9 July 2021).

2. Molinspiration. Calculation of Molecular Properties and Bioactivity Score. 2022. Available online: https://www.molinspiration. com/cgi-bin/properties (accessed on 9 July 2021).

3. Sharma, A.K.; Srivastava, G.N.; Roy, A.; Sharma, V.K. ToxiM: A Toxicity Prediction Tool for Small Molecules Developed Using Machine Learning and Chemoinformatics Approaches. Front. Pharmacol. 2017, 8, 880. [CrossRef] [PubMed]

4. Metabiosys. ToxiM, Toxicity Prediction Tool for Small Molecules. 2017. Available online: http://metagenomics.iiserb.ac.in/ ToxiM/index.html (accessed on 9 July 2021).

5. $\quad$ Banerjee, P.; Dunkel, M.; Kemmler, E.; Preissner, R. SuperCYPsPred-a web server for the prediction of cytochrome activity. Nucleic Acids Res. 2020, 48, W580-W585. [CrossRef] [PubMed]

6. Structural Bioinformatics Group. SuperCYPsPred, A Web Server for the Prediction of Cytochrome Activity. 2019. Available online: http:/ /insilico-cyp.charite.de/SuperCYPsPred/index.php?site=home\%20c (accessed on 9 July 2021).

7. Keiser, M.J.; Roth, B.L.; Armbruster, B.N.; Ernsberger, P.; Irwin, J.J.; Shoichet, B.K. Relating protein pharmacology by ligand chemistry. Nat. Biotechnol. 2007, 25, 197-206. [CrossRef] [PubMed]

8. UCSF, Shoichet Laboratory. Similarity Ensemble Approach (SEA). 2007. Available online: https://sea.bkslab.org/ (accessed on 9 July 2021). 